\title{
Ex vivo multiphoton analysis of rabbit corneal wound healing following conductive keratoplasty
}

Tsung-Jen Wang

Taipei Medical University Hospital

Department of Ophthalmology

Taipei 110, Taiwan

and

National Taiwan University Hospital

Department of Ophthalmology

Taipei 110, Taiwan and

National Taiwan University

College of Medicine

Taipei 100, Taiwan

\section{Wen Lo}

\section{Chiu Mei Hsueh}

National Taiwan University

Department of Physics

Taipei 106, Taiwan

Ming-Shium Hsieh

Department of Medicine

Taipei Medical Univeristy

Taipei 110, Taiwan

\section{Chen-Yuan Dong}

National Taiwan University

Department of Physics

Taipei 106, Taiwan

E-mail: cydong@phys.ntu.edu.tw

\section{Fung-Rong $\mathrm{Hu}$}

National Taiwan University Hospital

Department of Ophthalmology

Taipei 100, Taiwan

and

National Taiwan University

College of Medicine

Taipei 100, Taiwan

E-mail: fungronghu@ntu.edu.tw

\begin{abstract}
Ex vivo multiphoton imaging is used to characterize rabbit corneal wound healing after conductive keratoplasty (CK) procedures. $\mathrm{CK}$ is performed on the right eyes from eight New Zealand albino rabbits while the left eyes are punctured by a keratoplast tip without energy application. Rabbits are humanely sacrificed 1 day, 1, 2, and 4 weeks after the CK procedure. Eye balls are enucleated and placed on the microscope for multiphoton imaging. Multiphoton imaging reveals damage of corneal epithelium and stroma caused by the CK procedure and the subsequent wound healing process can be followed without histological procedures. Multiphoton excited autofluorescence images demonstrate that re-epithelilialization is accomplished within 1 week in both CK and control groups. However, epithelial hyperplasia is observed in CK corneas. In addition, stromal wounds in the control group become inconspicuous within 1 week while obvious wounds still exist in CK corneas for at least 4 weeks. Postconductive keratoplasty corneal damage and wound healing can be characterized by multiphoton microscopy without histological procedures. Our results suggest that multiphoton microscopy has potential in the clinical evaluation of corneal damage due to refractive surgery, and can be used to study and reduce the unwanted side effects of these procedures. $\odot 2008$ Society of Photo-Optical Instrumentation Engineers. [DOI: $10.1117 / 1.2943156]$
\end{abstract}

Keywords: corneal wound healing; conductive keratoplasty; collagen; multiphoton microscopy; second harmonic generation.

Paper 07188R received May 24, 2007; revised manuscript received Dec. 19, 2007; accepted for publication Dec. 19, 2007; published online Jul. 1, 2008.

\section{Introduction}

In refractive surgical procedures, techniques based on different physical mechanisms have proven to be effective in vision improvement. Commonly applied optical techniques such as photorefractive keratectomy (PRK), ${ }^{1,2}$ and laser in situ keratomileusis (LASIK), ${ }^{3,4}$ are invaluable for treating myopia. Clinical techniques for correcting hyperopia and presbyopia by shrinking the corneal periphery have been developed for more than a century. In recent years, the optothermal technique of laser thermal keratoplasty (LTK) ${ }^{5-7}$ was developed

Address all correspondence to Chen-Yuan Dong, Department of PhysicsNational Taiwan University Taipei, Taiwan 106 Taiwan; Tel: 8862-2-3366-5155; Fax: 886-2-2363-9984; E-mail: cydong@phys.ntu.edu.tw or Fung-Rong Hu, National Taiwan University Hospital and Collagen of Medicine, Taipei 100, Taiwan; Tel. 886-2-23123456, ext. 5189, 886-2-23412875; E-mail: fungronghu@ntu.edu.tw and more recently, the thermal technique conductive keratoplasty $(\mathrm{CK})$ was invented for treating hyperopia and presbyopia patients. ${ }^{8-10} \mathrm{CK}$ has been an effective, predictable, and stable technique to improve presbyopia due to aging. ${ }^{11-13}$

Conductive keratoplasty (CK) is a nonablative, radiofrequency-based, thermal treatment. In this procedure, radiofrequency current is delivered into the cornea through fine conducting tips (keratoplast tips) that are arranged at the corneal periphery. ${ }^{14,15}$ Due to the heat generated by passing a current through the cornea, collagen lamellae in the area surrounding the tip shrink in a controlled fashion to form a column of denatured collagen. ${ }^{16}$ The alteration of corneal architecture can then lead to vision correction for presbyopic and hyperopic patients. In this procedure, the immediate and long-

$1083-3668 / 2008 / 13(3) / 034019 / 9 / \$ 25.00$ ○ 2008 SPIE 
term effects of presobyopic and hyperopic correction depend on corneal curvature changes induced by collagen shrinkage. Despite the success of the $\mathrm{CK}$ procedure, regression issues remain to be addressed. The mechanism investigation of the postsurgical regression process may lead to improvement in the CK procedure and in providing care for patients.

To evaluate the corneal wound healing process after refractive surgery, conventional histopathological examination was used in earlier investigations. ${ }^{17}$ However, drawbacks associated with histological examinations limit the investigation of the dynamic processes of corneal wound healing. First, histological procedures require tissue removal and specimen processing that render in vivo observation of the dynamic events associated with corneal wound healing impossible. Furthermore, traditional histopathological examination has limited capacity in evaluating the architecture of collagen fiber that is the key element of wound healing after refractive corneal procedures. It is the limitations of the traditional histological approach and the prospect of developing a real-time, clinical diagnostic technique to evaluate the effects of refractive surgery procedures that prompted us to search for an alternative imaging modality in studying corneal wound healing. We found that multiphoton imaging is a technique that can bypass the disadvantages of histological examination.

Biological multiphoton microscopy based on two-photon fluorescence excitation was developed in the early 1990s as a new imaging technique with the advantages of enhanced depth discrimination, reduced photodamage, and increased penetration depth. ${ }^{18,19}$ Confocal-like image quality can be obtained from living cells and tissues without inducing detectable damage. ${ }^{20-22}$ In addition, $\operatorname{NADP}(\mathrm{H})$ autofluorescence (AF) within living cells can be detected through multiphoton microscopy. ${ }^{23}$ Furthermore, nonlinear polarization effects from a special class of biological materials is also of biomedical significance. Specifically, nonvanishing second-order susceptibility can contribute to second-harmonic generation (SHG) signals in biological structures lacking an inversion symmetry. ${ }^{24-27}$ To be specific, collagen and muscle fibers are biological materials known to be effective in generating second-harmonic signals. Since corneal stroma is composed mainly of type- 1 collagen, SHG is effective in the imaging of the stroma. ${ }^{28-30}$ Compared to conventional histological procedures, the unique advantage of multiphoton imaging in obtaining structural information of the corneal architecture renders this technique to be an exciting tool for the in vivo investigation of wound healing processes associated with refractive surgery procedures. Multiphoton microscopy offers the exciting prospect of becoming an ophthalmic imaging technique for evaluating the clinical outcome of refractive surgery. However, prior to in vivo and eventual clinical studies, ex vivo investigations must first be carried out to test the feasibility of multiphoton imaging in resolving corneal structural changes associated with these procedures.

The purpose of this study is to test such feasibility on postoperative ex vivo rabbit corneas after the CK procedures. We image and characterize the corneal wound healing process at four time points-day 1, and weeks 1,2, and 4-following the $\mathrm{CK}$ procedure.

\section{Materials and Methods}

Eight New Zealand albino rabbits weighing 1.5 to $2.0 \mathrm{~kg}$ were used in this study. The use of rabbits was approved by the Institute of Animal Care and Use Committee in the College of Medicine at National Taiwan University and conformed to the ARVO Statement for the Use of Animals in Ophthalmic and Vision Research. Each rabbit received intramuscular xylazine $(10 \mathrm{mg} / \mathrm{kg})$ and ketamine hydrochloride $(50 \mathrm{mg} / \mathrm{kg})$ for anesthesia. Tetracaine eyedrops were used for topical anesthesia.

\subsection{Conductive Keratoplasty System and Procedure}

The right eyes from eight New Zealand albino rabbits received conductive keratoplasty $(\mathrm{CK})$. To establish a control group, the left eyes were punctured with the same conducting probe without current delivery.

The ViewPoint CK system (Refractec, Incorporated, Irvine, California) was used in the CK procedure. After applying an eyelid speculum, the cornea was marked with a gentian violet-dampened, eight-intersection $\mathrm{CK}$ marker that marked the 7-mm optic zone (OZ).

Prior to applying the CK procedures, the corneal surface was dried with a fiber-free sponge to avoid dissipation of applied energy through the damp surface. The modified keratoplast tip, $300 \mu \mathrm{m}$ in length and $90 \mu \mathrm{m}$ in diameter, was then placed on the cornea at the treatment points and attempts were made to place the tips perpendicular to the corneal surface. Light pressure was then applied until the tip penetrated the stroma and the insulator stop was reached. Energy was then applied by depressing the foot pedal. All the right eyes received treatment at the setting of $350-\mathrm{kHz}$ radio frequency at $60 \%$ power (actual power $0.3 \mathrm{~W}$ ) for $0.6 \mathrm{~s}$. For the control group, the left eyes were punctured by the keratoplast tip without the application of thermal energy.

Two rabbits were sacrificed for observation at each time point i.e., day 1 , and weeks 1,2 , and 4 , postoperatively. The eyeballs excised from rabbit were placed in a container directly mounted on the microscope and immersed in phosphate buffered saline (PBS) solution for multiphoton observation. Water immersion objective was directly immersed in PBS to facilitate imaging of the curved ocular surface.

\subsection{Multiphoton Fluorescence and Second- Harmonic-Generation Microscope}

A homebuilt multiphoton autofluorescence (MAF) and SHG microscopic system was used in this study. The multiphoton laser scanning microscopic imaging system is similar to the one previously described. ${ }^{29-31}$ The imaging system is based on a commercial upright microscope (E800, Nikon, Japan). The output of a titanium-sapphire pulse laser (Tsunami, Spectra Physics, Mountain View, California) pumped by a diodepumped, solid state laser (Millennia X, Spectra Physics) operating at a wavelength of $780 \mathrm{~nm}$ was used as the excitation source.

For focusing the excitation laser and collecting the MAF and SHG signals from the cornea specimens, a water immersion objective (SFluor, WI, 40×, NA 0.8, Nikon) was used. The collected signals first pass through the short-pass, dichroic mirror used in reflecting the excitation source into the focusing objective (700dcspruv-3p, Chroma Technology, 


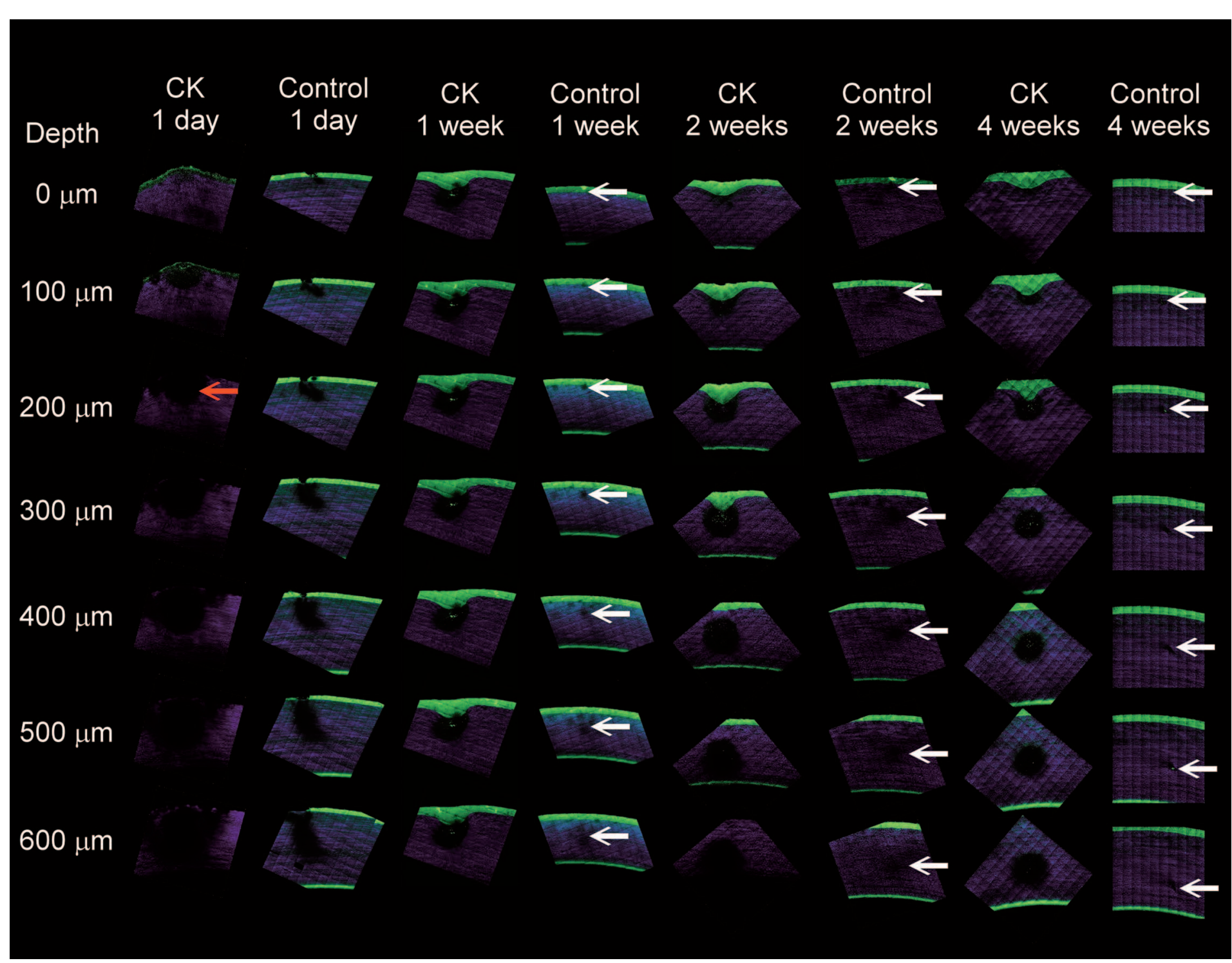

Fig. 1 The depth-dependent multiphoton images obtained after different timelapes for both CK and control corneas. At day 1 after surgery, epithelial removal is observed in both CK and control groups due to the mechanical injury caused by probe insertion. The re-epthelialization in both CK and control corneas are accomplished within one week. However, in the mulitphoton images from week 1 to week 4, epithelial hyperplasia is observed in CK corneas, while the epithelia in the control group remain normal. In addition, SHG imaging demonstrates that, compared to purely mechanical injury in control groups, currents cause larger and deeper stromal damage in CK corneas. Within the control group, the wound area caused by keratoplasty tip insertion is more evident at day 1 and becomes obscure within one week. Nevertheless, structural alternation (indicated by white arrows) in corneal stroma are detectable in the image series along depth. (Blue: second harmonic generation; green: autofluorescence.) 

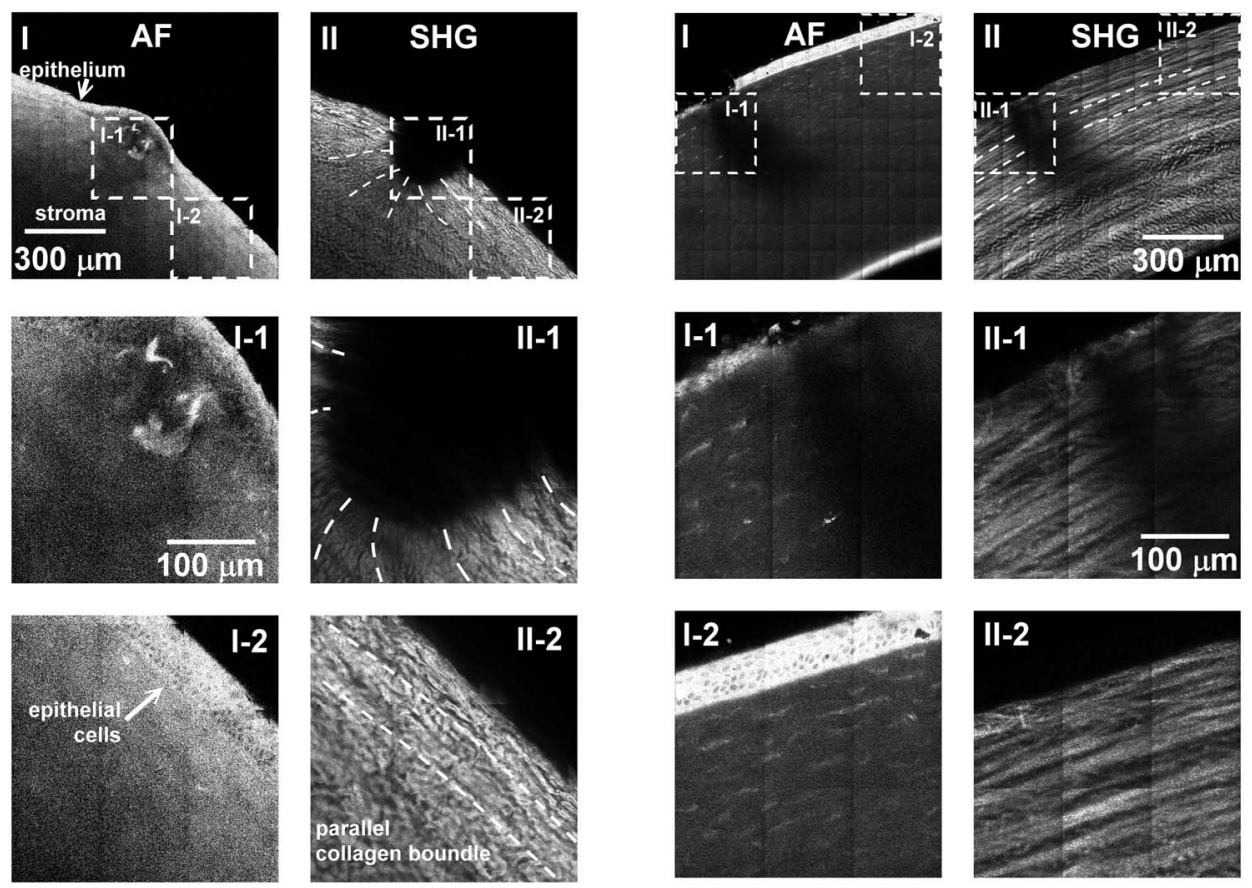

Fig. 2 Day 1 results. (a) CK cornea. The AF image is shown in I and SHG signal was shown in II. Magnified images from selected regions with areas 1 and 2 are further analyzed in boxes I-1, I-2. II-1, and II-2. I, the epithelium and stroma in the wound (I-1) and nearby regions (I-2) were visible. The radial pattern of collagen toward the wound was noted and marked with dotted arcuate lines. The trend was more obvious in higher magnification (II-1), and the arrangement of collagen fiber in the nearby region around the stromal wound was not affected (II-2). (b) Control cornea. The damage caused by the keratoplast tip in the epithelium and stroma can be respectively detected by the loss of autofluorescent cells (I) and SHG collagen fibers (II).

Rockingham, (Varment). The broadband MF and SHG are separated by a secondary dichroic mirror (435 dcxr, Chroma Technology) and respectively filtered by two bandpass filters (MF: E435lp-700sp, SHG: HQ390/20, Chroma Technology) before reaching the detectors. For detecting the autoflourescence (AF) and SHG signals, photon-counting photomultiplier tubes (R7400P, Hamamatsu, Japan) were used.

\section{Results}

\subsection{Depth-Dependent Multiphoton Images at Different Observation Time}

To appreciate the ability of multiphoton imaging in characterizing the effects of the $\mathrm{CK}$ procedure, representative MAF (green) and SHG (blue) images of the ex vivo rabbit corneas scanned along different depths and at different postoperative times are shown in Fig. 1. Images of the control corneas are also shown for comparison, and all images were oriented in the same fashion with the epithelium side up and endothelium side down. In all images, the CK site (red arrow in the day $1,200-\mu \mathrm{m}$ image) can be visualized by the region of the stroma devoid of SHG signal. The epithelial wounding caused by mechanical injury can be visualized by multiphoton excited autofluorescence in both $\mathrm{CK}$ and the control group. The re-epithelialization is accomplished within 1 week in both groups. The epithelia at wounding sites in the control group recovered after 1 week of recovery. However, epithelial hyperplasia is observed at the wounding sites of CK corneas. The epithelial hyperplasia lasts at least 4 weeks after surgery. Furthermore, SHG imaging shows structural alternation of postsurgical corneal stroma in both the $\mathrm{CK}$ and control groups. Compared to the control group, deeper and wider damaged regions in the stroma are found in CK corneas. Regions devoid of SHG signals remain evident 4 weeks following the CK procedure. For the control group corneas, the disruption of the epithelium and stroma is indicated by the respective lack of autofluorescent cells and SHG signal, and is most prominent in the day 1 cornea. Except for a region within the stroma devoid of the SHG signal, the affected wound areas appear to heal quickly and remain almost unnoticeable after the first week. The white arrows in Fig. 1 indicate the inconspicuous stromal structure alternation in the control group along the depth.

\subsection{Detailed Examination of Conductive- Keratoplasty-Operated Corneas}

To understand how the CK procedure alters stromal architecture on a larger scale, large area MAF and SHG images are shown in I and II in Figs. 2-5. Multiphoton imaging provides submicron information of cells and extracellular matrices in corneal stroma. To appreciate this ability, selected regions of interest (ROI) are magnified and shown in I-1, I-2. II-1 and II-2 in Figs. 2-5. The large-area multiphoton scans of rabbit cornea in the $\mathrm{CK}$ and control groups at the four time points and acquired at the imaging depth of $200 \mu \mathrm{m}$ are respectively shown in parts (a) and (b) of each figure. In addition, the separated MAF and SHG images of each dataset are shown in boxes 1 and 2, respectively. In both the MAF and SHG images, we chose two ROIs for magnification. In the case of 
$\mathrm{AF}$
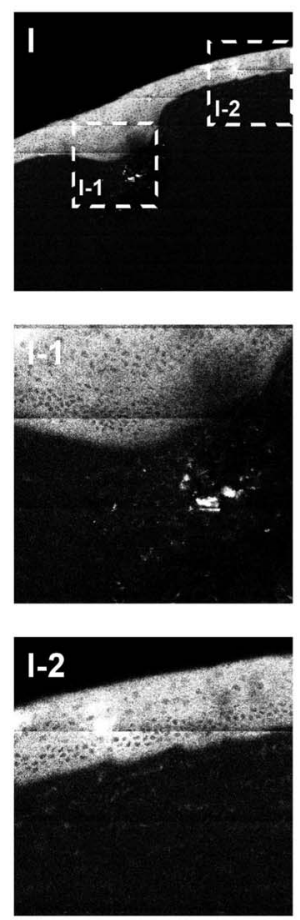

SHG
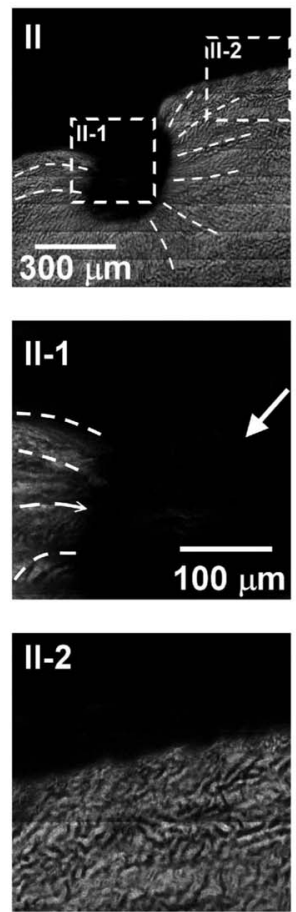

(a)
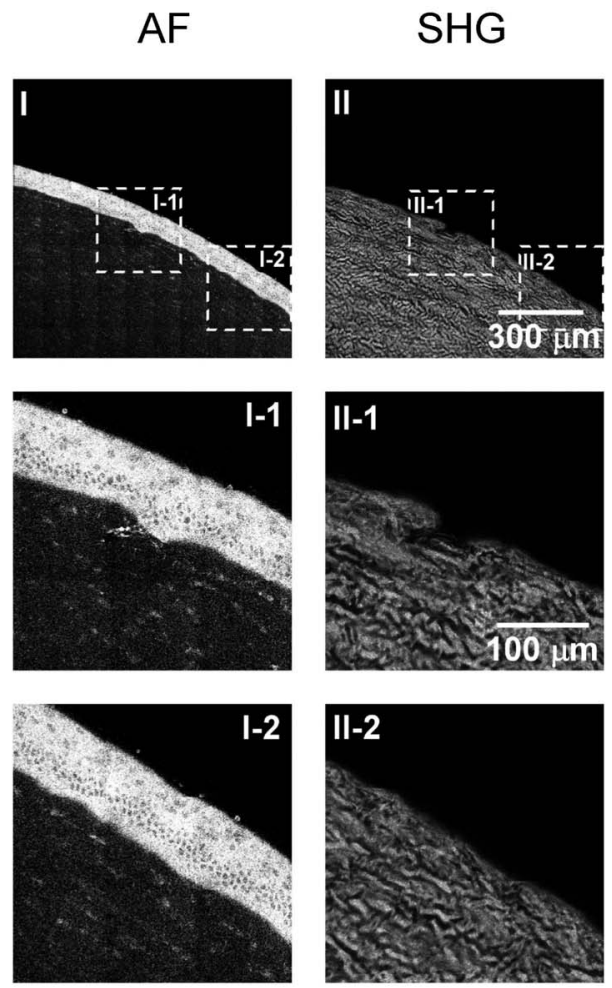

(b)

Fig. 3 Week 1 results. (a) CK cornea. In I, epithelial hyperplasia was found at the wound area. The numbers of layers increased (box 1-1), and the numbers of epithelial layers at regions away from the CK site remained normal (I-2). In II, the radial pattern of collagen surrounding the wound still existed. The general pattern was marked with dotted arcuate lines. In higher magnification, the radial pattern of collagen surrounding the wound is visualized with me SHG signals detected in the stromal wound area (II-1). The arrangement of collagen fiber in the nearby region remains in parallel fashion (II-2). (b) Control cornea. In 1, the epithelium almost healed without obvious damage. The epithelial wound area returned to a normal epithelial lamellar pattern without epithelial hyperplasia (I-1). The same is observed with the epithelium away from the wound (box 1-2). In 2, arrangement of collagen fibers was not affected. In the higher magnification image, the parallel pattern of collagen bundles was observed (II-1, II-2).

MAF images, the two areas represent the epithelial area where the keratoplast tip was applied (I-1) and a nearby epithelial region away from the wound (I-2). For SHG stromal imaging, both the wound region (II-1), and a nearby region (II-2) are shown for comparison.

\subsection{Day 1}

\subsubsection{Conductive keratoplasty cornea}

A representative multiphoton image of the CK cornea acquired at the imaging depth of $200 \mu \mathrm{m}$ is shown in Fig. 2(a). In the operated cornea, damaged epithelium can be detected by AF imaging [Fig. 2(a)] and it was observed that the application of CK resulted in localized corneal protrusion. In addition, SHG imaging reveals a radial pattern of collagen surrounding the wound [Fig. 2(a), II and II-1]. However, the swelling was not observed immediately adjacent to the wound area [Fig. 2(a), boxes I-2 and II-2], and round epithelial cells typical of normal epithelial tissue can be observed. In comparison, the SHG signal was useful in revealing the radial pattern of collagen surrounding the wound. The contraction trend was shown to be directed toward the wound applied by the keratoplast tip [Fig. 2(a) II] and is indicated by the dotted, arcuate lines in the enlarged image [Fig. 2(a), II-1]. Collagen fiber arrangement in the nearby region appears to be unaffected as parallel collagen lamellae are observed [Fig 2(a), II-2].

\subsubsection{Control cornea}

In comparison, the control group corneas are affected differently [Fig. 2(b)]. While regions of the autofluorescent epithelium have been removed [Fig. 2(b), I], the localized corneal swelling observed in the CK case was not seen. In addition, although the SHG images showed regions void of the secondharmonic signal, presumably due to the physical damage caused by the tip, the layered corneal collagen architecture remained intact. Furthermore, no radial pattern of collagen was noticed surrounding the wound [Fig. 2(b), II, II-1 and II-2]. The size of the stromal wound seems only due to the tip intrusion and is smaller than that found in the CK cornea. Therefore, without histological procedures, multiphoton imaging can be used to reveal structural alterations in CK corneas. In particular, SHG imaging indicates that corneal stroma damage is localized and that comparison with previous studies $^{27}$ on the thermal effects of porcine corneas suggest that loss of SHG signal at the CK site is caused by localized temperature increase exceeding $80^{\circ} \mathrm{C}$. 

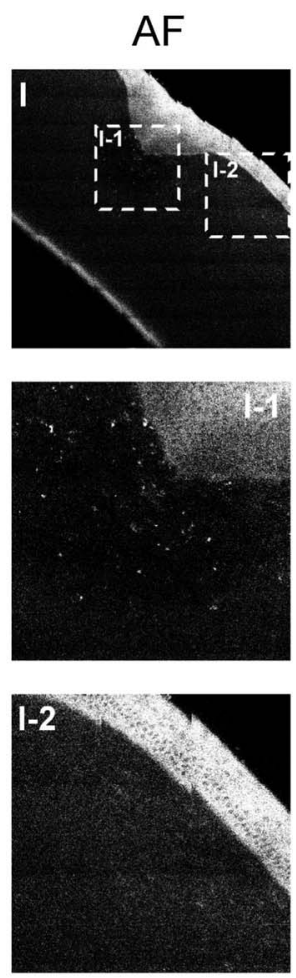
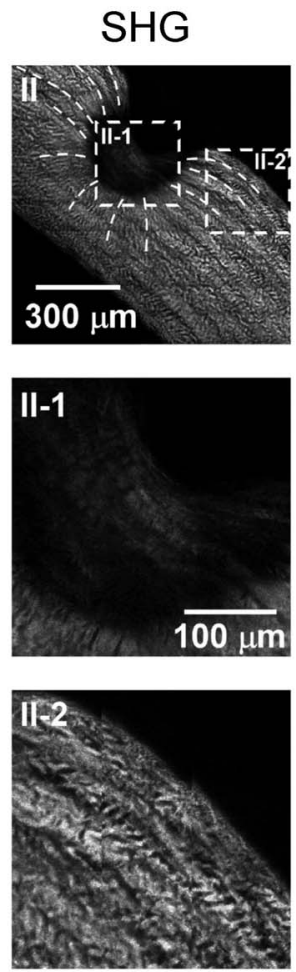

(a)
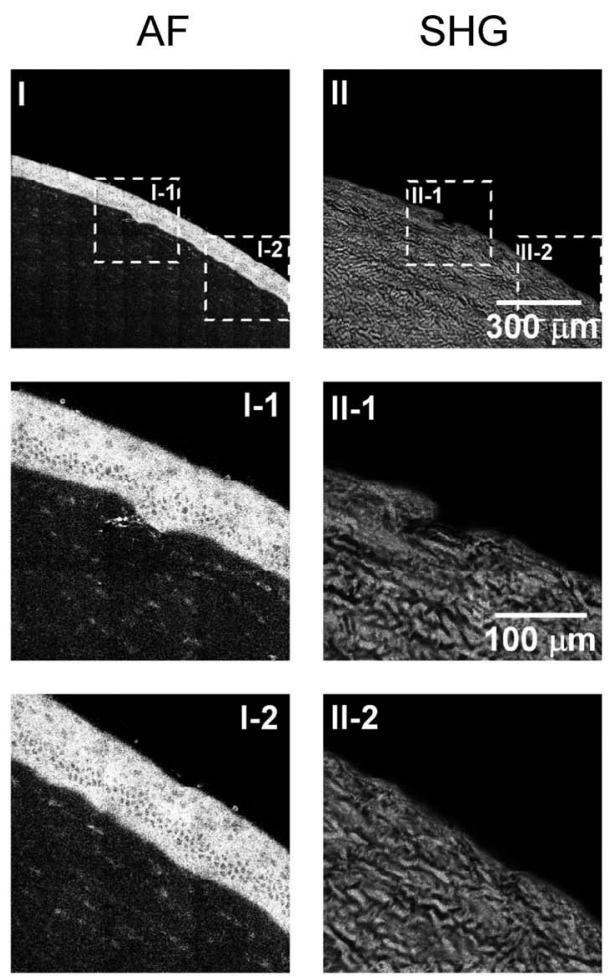

(b)

Fig. 4 Week 2 results. (a) CK cornea. In 1, epithelial hyperplasia was found at the wound. The numbers of layers increased at the epithelial wound area (box I-1), and the numbers of layers at nearby regional areas remained normal (I-2). In II, the radial pattern of collagen surrounding the wound still exists. This trend was marked with dotted arcuate lines. The regenerated SHG signal within the wound became more prominent (II-1). The parallel collagen lamellae was not affected away from the stromal wound (II-2). (b) Control cornea. Except for the lack of SHG signal at the insertion point, both the epithelium and stroma have their normal appearance. (II and II-1).

\subsection{Week 1}

\subsubsection{Conductive keratoplasty cornea}

Several prominent features were found in the CK cornea one week postoperation. First, epithelial hyperplasia was found at the wounding site with the epithelial layers growing into the wound [Fig. 3(a), I]. The radial pattern of collagen surrounding the wound is still present [Fig. 3(a), II and II-1], and the numbers of epithelial layers increased at the wound area [Fig. 3(a), I-1]. However, the epithelial tissue at the nearby region remained unaffected [Fig. 3(a), II-2]. At this point, no SHG signals were found in the stromal wound area [Fig. 3(a), II-1], and the orthogonal arrangement of collagen fiber in nearby regions appears to be unaffected [Fig. 3(a), II-2].

\subsubsection{Control cornea}

The corneal epithelium has returned to the normal lamellar structure without signs of epithelial hyperplasia [Fig. 3(b), boxes 1 and 1-1]. Comparison with nearby epithelium reveals an almost identical structural motif [Fig. 3(b), I-2]. Within the stroma, the radial pattern of collagen surrounding the wound, characteristic of CK corneas, is absent. However, a region with reduced SHG signal is observed [Fig. 3(b), II and II-1]. Comparison with the depth-resolved images in Fig. 1 suggests that this region corresponds to the tip insertion point. Overall, the arrangement of collagen fiber in the area at the stromal wound and a nearby region [Fig. 3(b), II-2] is remarkably similar. These observations suggest that the wound healed well in control eyes by the end of the first week.

\subsection{Week 2}

\subsubsection{Conductive keratoplasty cornea}

In the CK cornea, epithelial hyperplasia was found at the CK site as the inward growth of epithelium persisted [Fig. 4(a), I]. The radial pattern of collagen surrounding the wound remained as SHG signal was detected in the stromal wound site [Fig. 4(a), II]. The numbers of epithelial layers increased at the epithelial wound area [Fig. 4(a), I-1], and the numbers of epithelial layers at a nearby region remained unaffected [Fig. 4(a), I-2]. The most prominent feature of this sample is the detection of SHG signal in the stromal wound area [Fig. 4(a), II-1]. In this specimen, the contour of the SHG signal lies along the general direction of the underlying stromal lamellae, indicating that the collagen architecture damaged by the $\mathrm{CK}$ procedure has begun to be regenerated. The arrangement of collagen fiber in a nearby region was not affected, and the lamellar structure resembles that found in normal stroma [Fig. 4(a), II-2].

\subsubsection{Control cornea}

The multiphoton imaging results of the control eye at week 2 were similar to that found at week 1. As Fig. 4(b) shows, the 

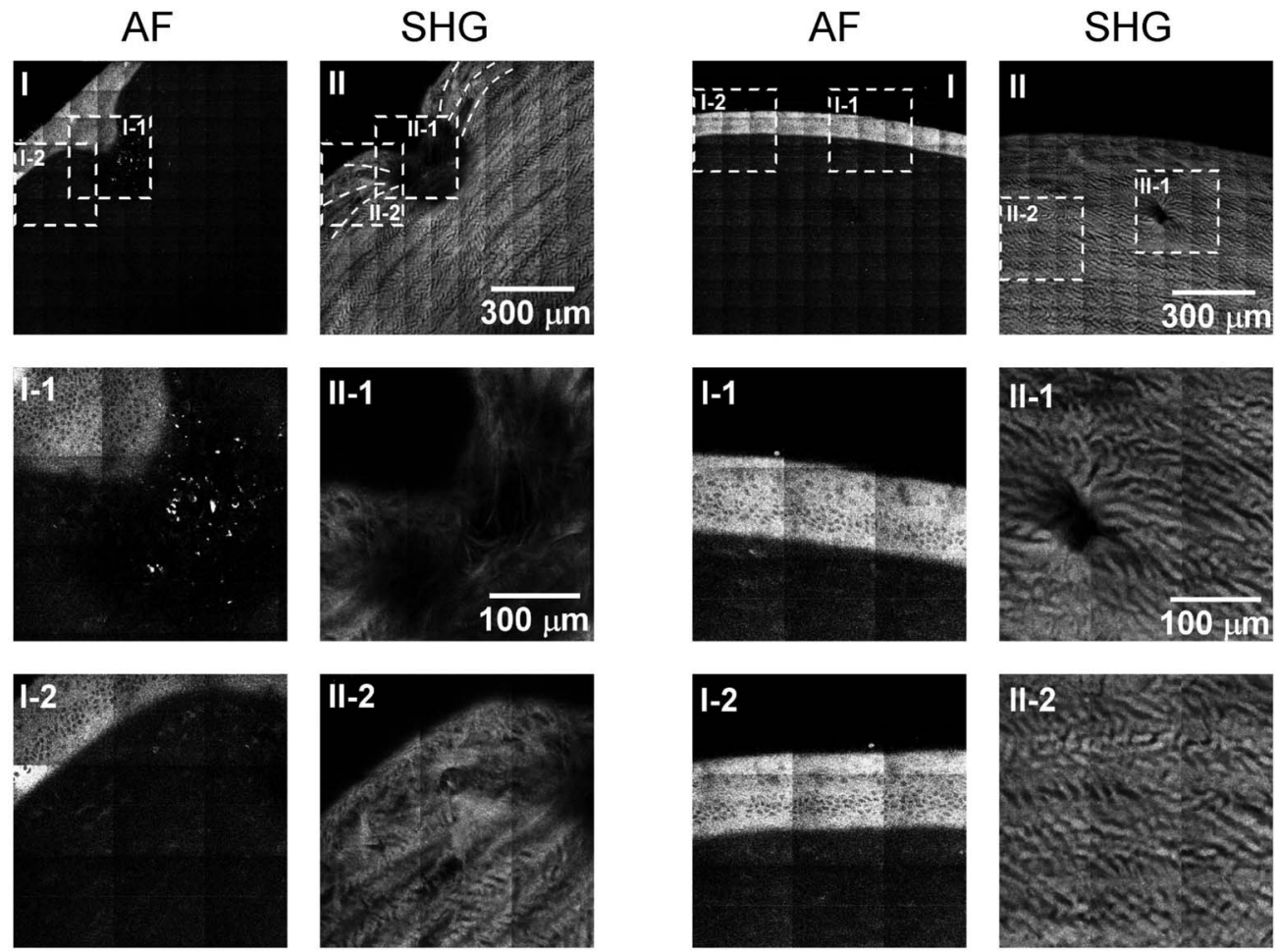

(a)
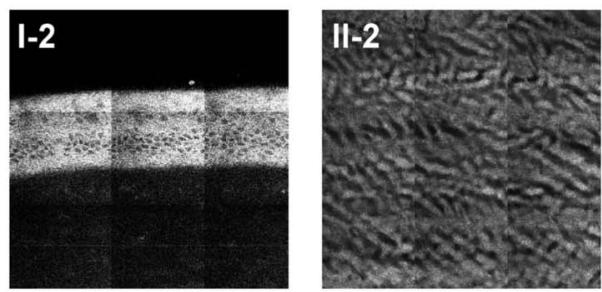

(b)

Fig. 5 Week 4 results. (a) CK cornea. In I, epithelial hyperplasia persists at the wound area. The number of epithelial layers remained increased (I-1), and those at nearby regions remained normal (I-2). In 2, the wound seems more contracted compared with the previous observation times. The regenerated SHG signal can be detected (II-1). The collagen bundles remained parallel away from stromal wound (II-2). (b) Control cornea. Except for the lack of SHG signal at the insertion point, both the epithelium and stroma have their normal appearance (I and II).

only noticeable difference from the normal part of the cornea is a localized region of perturbed SHG structure. Most likely, that corresponds to the tip insertion point.

\subsection{Week 4}

\subsubsection{Conductive keratoplasty cornea}

In the CK case, epithelial hyperplasia persisted at the wound area [Fig. 5(a), I]. The CK damage in the stromal wound area remained prominent [Fig. 5(a), II]. The increase in the numbers of epithelial layers persisted at the epithelial wound area [Fig. 5(a), I-1], and that at a nearby epithelial region appears to remain normal [Fig. 5(a), I-2]. The collagen contraction pattern at the wound remained obvious. Similar to week 2 results, SHG imaging revealed that the wound region was occupied by newly synthesized collagen fibers or was due to resumption of collagen architecture of original damaged collagen fibers [Fig. 5(a), II-1]. In comparison, the arrangement of collagen fiber in the area around the stromal wound remained unaffected [Fig. 5(a), II-2].

\subsubsection{Control Cornea}

Multiphoton imaging results of the week 4 control cornea are remarkably similar to that of the week 2 specimen. The most prominent feature is the presence of a region within the stroma where SHG imaging reveals perturbed collagen fiber structures. Most likely, that region corresponds to the tip in- sertion point [Fig. 5(b), II and II-1]. Otherwise, the structure of the cornea is virtually identical to that found in a nearby region [Fig. 5(b), II-2].

\section{Discussion}

The relatively new procedure of conductive keratoplasty is a local thermal procedure induced by radio-frequency energy delivered directly to the treatment spots of corneal stroma. ${ }^{32}$ The thermal energy used to modify the corneal structure was not produced by the probe but is caused by resistive heating from current flow. ${ }^{11-13}$ Compared to noncontact LTK, in which the application spots were processed simultaneously, the CK procedure delivers thermal energy in a sequence of paired and opposing spots. A circle of treated spots making concentric rings at the mid-periphery of the cornea caused the corneal periphery to shrink, leading to a steepened cornea center. The effects of CK application at the corneal midperiphery can correct low hyperopia and presbyopia in middle-aged and elderly patients with minimal complications. Therefore, the focal distance is reduced and the patient's near vision can also be improved. In other applications, CK was also reported to be able to treat irregular astigmatism in keratoconus patients for vision improvement. ${ }^{32}$

The use of multiphoton fluorescence (MF) and SHG microscopy for normal corneal imaging has the advantages of bypassing tissue processing procedures. ${ }^{29,33}$ The characteristics and properties of the nonlinear fluorescence excitation 
and the SHG from collagen enables the visualization of subcellular corneal structures. Pathological corneal change in conditions such as keratoconus has also been demonstrated with the use of MAF and SHG microscopy. ${ }^{31}$

Since conductive keratoplasty modifies corneal stromal collagen in changing the corneal curvature, the combination of MF and SHG miscroscopy may be a satisfactory technique to evaluate this procedure with minimal tissue invasion. In our study, detailed structural alterations of corneas after conductive keratoplasty were demonstrated at different time points. The immediate changes of corneal epithelium and stroma after the procedure were the combinations of physical effect and thermal effect of the keratoplast tip. The observed damage is more significant in CK corneas than in the control cases. The modified, radial pattern of collagen fiber bundles appeared immediately after the CK procedure. This effect persisted 4 weeks after surgery, and the radial patterns seem to be unaffected by collagen regeneration at the wounding site. As for corneal epithelium, AF imaging was able to reveal the tissue damage by the keratoplast tip. The corneal epithelium was restored in the CK eyes. However, epithelial hyperplasia was shown to be an inevitable effect of the CK procedure. The hyperplasic phenomenon existed until four weeks after the CK procedure and was not observed in the control eye.

\section{Conclusions}

By the use of the combined imaging modality of multiphoton autofluorescence imaging and second-harmonic-generation microscopy, we demonstrate that structural alterations and the subsequent wound healing due to conductive keratoplasty may be followed in ex vivo rabbit corneas without the addition of extrinsic probes. The CK procedure causes immediate structural alteration of the epithelium and stroma. The changes to epithelium and stroma occur immediately. Following the $\mathrm{CK}$ procedure, re-epithelialization is noticed and is accompanied by compensatory epithelial hyperplasia. The stromal wound contracts at first, and then the degree of contraction changes at different time points. The collagen regeneration or reorganization was noticeable at week 2 is found to be similar to that found 4 weeks after surgery, suggesting that the regeneration or reorganization process stabilizes after 2 weeks.

The limited invasion of multiphoton imaging provides the feasibility of in vivo imaging with multiphoton techniques. However, prior to approaching in vivo examination, ex vivo imaging is still indispensible in establishing background knowledge. Our results demonstrate that multiphoton imaging effectively indicates structure alteration in corneal stroma after refractive surgery with minimal invasion. Our multiphoton results also suggest that $\mathrm{CK}$ is a relatively safe procedure without substantial perturbation to the corneal epithelium. With additional development, the applications of multiphoton imaging in the clinical evaluation of corneal wound healing after refractive corneal procedures may be achieved in the future.

\section{Acknowledgments}

Financial support was provided by the Taipei Medical University Hospital Research Grant (Grant Number: 97TMUTMUH-10), National Taiwan University Hospital Research
Grant (Grant Number 96-S583) and National Science Council in Taiwan. This project was completed in the Optical Molecular Imaging Microscopy Core Facility (A5) of the National Genomic Project for Genomic Medicine (NRPGM) of the National Science Council in Taiwan.

\section{References}

1. J. Pietila, P. Makinen, S. Pajari, and H. Uusitalo, "Excimer laser photorefractive keratectomy for hyperopia," J. Refract. Surg. 13(6), 504-510 (1997).

2. P. Vinciguerra, D. Epstein, P. Radice, and M. Azzolini, "Long-term results of photorefractive keratectomy for hyperopia and hyperopic astigmatism," J. Refract. Surg. 14(2), S183-S185 (1998).

3. C. J. Argento and M. J. Cosentino, "Laser in situ keratomileusis for hyperopia," J. Cataract Refractive Surg. 24(8), 1050-1058 (1998).

4. K. Ditzen, H. Huschka, and S. Pieger, "Laser in situ keratomileusis for hyperopia," J. Cataract Refractive Surg. 24(1), 42-47 (1998).

5. C. A. Eggink, Y. Bardak, M. H. M. Cuypers, and A. F. Deutman, "Treatment of hyperopia with contact Ho: YAG laser thermal keratoplasty," J. Refract. Surg. 15(1), 16-22 (1999).

6. D. D. Koch, A. Abarca, R. Villarreal, R. Menefee, T. Kohnen, A. Vassiliadis, and M. Berry, "Hyperopia correction by noncontact holmium:YAG laser thermal keratoplasty clinical study with two-year follow-up," Ophthalmology 103(5), 731-740 (1996).

7. A. C. Neumann, D. Sanders, M. Raanan, and M. Deluca, "Hyperopic thermokeratoplasty-clinical-evaluation," J. Cataract Refractive Surg. 17(6), 830-838 (1991).

8. D. Y. Lin and E. E. Manche, "Two-year results of conductive keratoplasty (CK) for the correction of low to moderate hyperopia," Invest. Ophthalmol. Visual Sci. 44, U27-U27 (2003).

9. I. G. Pallikaris, T. L. Naoumidi, and N. I. Astyrakakis, "Conductive keratoplasty to correct hyperopic astigmatism," J. Refract. Surg. 19(4), 425-432 (2003)

10. I. G. Pallikaris, T. L. Naoumidi, S. I. Panagopoulou, A. K. Alegakis, and N. I. Astyrakakis, "Conductive keratoplasty for low to moderate hyperopia: 1-year results,” J. Refract. Surg. 19(5), 496-506 (2003).

11. M. B. McDonald, J. Davidorf, R. K. Maloney, E. E. Manche, and P. Hersh, "Conductive keratoplasty for the correction of low to moderate hyperopia-1-year results on the first 54 eyes," Ophthalmology 109(4), 637-649 (2002).

12. M. B. McDonald, D. Durrie, P. Asbell, R. Maloney, and L. Nichamin, "Treatment of presbyopia with conductive keratoplasty (R) - Sixmonth results of the 1-year United States FDA clinical trial," Cornea 23(7), 661-668 (2004).

13. M. B. McDonald, P. S. Hersh, E. E. Manche, R. K. Maloney, J. Davidorf, M. Sabry, and C. K. U. Investi, "Conductive keratoplasty for the correction of low to moderate hyperopia: U.S. clinical trial 1-year results on 355 eyes," Ophthalmology 109(11), 1978-1989 (2002).

14. W. W. Haw and E. E. Manche, "Conductive keratoplasty and laser thermal keratoplasty," Int. Ophthalmol. Clin. 42(4), 99-106 (2002).

15. B. Huang, "Update on nonexcimer laser refractive surgery technique: conductive keratoplasty," Curr. Opin. Ophthalmol. 14(4), 203-206 (2003).

16. E. Sporl, U. Genth, K. Schmalfuss, and T. Seiler, "Thermomechanical behavior of the cornea," Ger. J. Ophthalmol. 5(6), 322-327 (1996).

17. D. G. Dawson, H. F. Edelhauser, and H. E. Grossniklaus, "Long-term histopathologic findings in human corneal wounds after refractive surgical procedures," Am. J. Ophthalmol. 139(1), 168-178 (2005).

18. W. Denk, J. H. Strickler, and W. W. Webb, "Two-photon laser scanning fluorescence microscopy," Science 248(4951), 73-76 (1990).

19. P. T. So, C. Y. Dong, B. R. Masters, and K. M. Berland, "Two-photon excitation fluorescence microscopy," Апnи. Rev. Biomed. Eng. 2, 399-429 (2000).

20. B. R. Masters, P. T. So, and E. Gratton, "Multiphoton excitation fluorescence microscopy and spectroscopy of in vivo human skin," Biophys. J. 72(6), 2405-2412 (1997).

21. M. J. Miller, S. H. Wei, I. Parker, and M. D. Cahalan, "Two-photon imaging of lymphocyte motility and antigen response in intact lymph node," Science 296(5574), 1869-1873 (2002). 
22. Y. Sun, J. W. Su, W. Lo, S. J. Lin, S. H. Jee, and C. Y. Dong, "Multiphoton polarization imaging of the stratum corneum and the dermis in ex vivo human skin," Opt. Express 11(25), 3377-3384 (2003).

23. D. W. Piston, B. R. Masters, and W. W. Webb, "3-dimensionally resolved $\operatorname{Nad}(\mathrm{P}) \mathrm{H}$ cellular metabolic redox imaging of the in situ cornea with 2-photon excitation laser-scanning microscopy," J. Microsc. 178, 20-27 (1995).

24. P. J. Campagnola and L. M. Loew, "Second-harmonic imaging microscopy for visualizing biomolecular arrays in cells, tissues and organisms," Nat. Biotechnol. 21(11), 1356-1360 (2003).

25. S. V. Plotnikov, A. C. Millard, P. J. Campagnola, and W. A. Mohler, "Characterization of the myosin-based source for second-harmonic generation from muscle sarcomeres," Biophys. J. 90(2), 693-703 (2006).

26. A. Zoumi, X. A. Lu, G. S. Kassab, and B. J. Tromberg, "Imaging coronary artery microstructure using second-harmonic and twophoton fluorescence microscopy," Biophys. J. 87(4), 2778-2786 (2004).

27. A. Zoumi, A. Yeh, and B. J. Tromberg, "Imaging cells and extracellular matrix in vivo by using second-harmonic generation and twophoton excited fluorescence," Proc. Natl. Acad. Sci. U.S.A. 99(17), 11014-11019 (2002).

28. S. J. Lin, R. J. Wu, H. Y. Tan, W. Lo, W. C. Lin, T. H. Young, C. J.
Hsu, J. S. Chen, S. H. Jee, and C. Y. Dong, "Evaluating cutaneous photoaging by use of multiphoton fluorescence and second-harmonic generation microscopy," Opt. Lett. 30(17), 2275-2277 (2005).

29. H. Y. Tan, S. W. Teng, W. Lo, W. C. Lin, S. J. Lin, S. H. Jee, and C. Y. Dong, "Characterizing the thermally induced structural changes to intact porcine eye, part 1: second harmonic generation imaging of cornea stroma," J. Biomed. Opt. 10, 054019 (2005).

30. S. W. Teng, H. Y. Tan, J. L. Peng, H. H. Lin, K. H. Kim, W. Lo, Y. Sun, W. C. Lin, S. J. Lin, S. H. Jee, P. T. C. So, and C. Y. Dong, "Multiphoton autofluorescence and second-harmonic generation imaging of the ex vivo porcine eye," Invest. Ophthalmol. Visual Sci. 47(3), 1216-1224 (2006).

31. H. Y. Tan, Y. Sun, W. Lo, S. J. Lin, C. H. Hsiao, Y. F. Chen, S. C. M. Huang, W. C. Lin, S. H. Jee, H. S. Yu, and C. Y. Dong, "Multiphoton fluorescence and second harmonic generation imaging of the structural alterations in keratoconus ex vivo," Invest. Ophthalmol. Visual Sci. 47(12), 5251-5259 (2006).

32. J. L. Alio, P. J. Claramonte, A. Caliz, and M. I. Ramzy, "Corneal modeling of keratoconus by conductive keratoplasty," J. Cataract Refractive Surg. 31(1), 190-197 (2005).

33. A. T. Yeh, N. Nassif, A. Zoumi, and B. J. Tromberg, "Selective corneal imaging using combined second-harmonic generation and two-photon excited fluorescence," Opt. Lett. 27(23), 2082-2084 (2002). 